TITLE:

\title{
Histone core slips along DNA and prefers positioning at the chain end
}

$\operatorname{AUTHOR}(S)$ :

Sakaue, T; Yoshikawa, K; Yoshimura, SH; Takeyasu, $\mathrm{K}$

\section{CITATION:}

Sakaue, T ...[et al]. Histone core slips along DNA and prefers positioning at the chain end. PHYSICAL REVIEW LETTERS 2001, 87(7): 078105.

ISSUE DATE:

2001-08-13

URL:

http://hdl.handle.net/2433/49894

RIGHT:

Copyright 2001 American Physical Society 


\title{
Histone Core Slips along DNA and Prefers Positioning at the Chain End
}

\author{
Takahiro Sakaue and Kenichi Yoshikawa \\ Department of Physics, Graduate School of Science, Kyoto University, Kyoto 606-8504, Japan
}

Shige H. Yoshimura and Kunio Takeyasu

Graduate School of Biostudies, Kyoto University, Kyoto 606-8502, Japan

(Received 18 December 2000; published 30 July 2001)

\begin{abstract}
We studied the stability and dynamics of a model of a nucleosome, the fundamental unit for the packing of long DNA in eukaryotes, using a Brownian dynamics simulation. For the proper folding of a stiff polymer on a core particle, moderate attractive interaction is shown to be essentially important, which explains the empirical experimental protocol for the reconstitution of nucleosomes. The effect of the chain end on the positioning of the core particle is examined and compared with the experimental data by atomic force microscopy measurement. It is also suggested that the core particle exhibits sliding motion along the chain as a manifestation of Brownian motion.
\end{abstract}

DOI: $10.1103 /$ PhysRevLett.87.078105

PACS numbers: $87.15 . \mathrm{He}, 61.25 . \mathrm{Hq}, 87.14 . \mathrm{Gg}$

Genomic DNA in eukaryotes is compactly folded into chromatin through several hierarchical packings, and the fundamental unit of the packing is called a nucleosome. One nucleosome consists of a histone octamer (two molecules of each of H2A, H2B, H3, and H4) and $146 \mathrm{bp}$ of DNA. The negatively charged stiff polymer wraps around a cationic protein core about two turns in a counterclockwise fashion [1-3]. It is expected that the manner of packing and the dynamics of nucleosomes are associated with gene activity in living cells. On DNA replication and transcription in eukaryotes, tight packing of DNA with a stable nucleosome structure sterically hinders the initial complex formation and the following activity of replication and transcription machinery. Many biochemical and molecular biological experiments have suggested that nucleosomes might be remodeled to relaxed or unfolded structures before the initiation of such events [1]. Theoretically, the effect of stiffness and/or electrostatic interaction on the equilibrium behavior of the binding between a polymer chain and a core particle have been reported [4-9]. These studies showed important aspects such as a wrapping transition and overcharging of a macroion by an oppositely charged polyelectrolyte, and possible applications to nucleosomes were considered. Unfortunately, the kinetic effect on the wrapping process seems to have been ignored in these studies.

The purpose of this Letter is to get a better understanding of not only static but also dynamic aspects of nucleosomes. We performed a Brownian dynamics simulation [10] using a simplified model which consists of a stiff homopolymer chain and a spherical core particle. A polymer chain is modeled by $N$ spherical monomers connected by bonds. We adopted $N=40$ in this study, which corresponds to a DNA chain of about $240 \mathrm{bp}$. The self-avoiding effect of the polymer chain is considered through the repulsive part of the Morse potential,

$$
U_{\text {M.rep }}=\epsilon_{m} T \sum \exp \left\{-\alpha_{m}\left(r_{i, j}-\sigma_{m}\right)\right\},
$$

where $\epsilon_{m}=0.2$ and $\alpha_{m}=24$. Thus, the radius of the monomer is approximately $0.5 \sigma_{m}$. We set the Boltzmann constant $k_{B}$ to unity throughout this article. The effective interaction between each monomer and core particle is modeled through the Morse potential,

$$
\begin{aligned}
U_{M}=\epsilon T \sum & {\left[\exp \left\{-2 \alpha\left(r_{i}-\sigma\right)\right\}\right.} \\
& \left.-2 \exp \left\{-\alpha\left(r_{i}-\sigma\right)\right\}\right],
\end{aligned}
$$

where $\alpha=6$ and $\sigma=1.9 \sigma_{m}$, so that the radius of the core particle is approximately $1.3 \sigma_{m}$. It is noted that this effective interaction is a parameter depending on the salt concentration, where larger $\epsilon$ corresponds to the experimental condition at low salt concentration. The radius of the monomer $0.5 \sigma_{m}$ and core particle $1.3 \sigma_{m}$ correspond to the volume of a cylindrical DNA segment with a radius of $1 \mathrm{~nm}$ and a disk-shaped histone core about $5 \mathrm{~nm}$ thick and with a radius of $3 \sim 3.5 \mathrm{~nm}$, respectively. Thus, the volume ratio between the core particle and polymer chain is almost the same as in a real nucleosome. The bonds between neighboring monomers are maintained by harmonic bonding potential,

$$
U_{\text {bond }}=\frac{k T}{2 \sigma_{m}^{2}} \sum\left(\left|\boldsymbol{r}_{i}-\boldsymbol{r}_{i+1}\right|-\sigma_{m}\right)^{2},
$$

where we choose a relatively large spring constant $k=$ 400 to keep the bond length at a nearly constant value $\sigma_{m}$. To model the chain stiffness, the following bending potential is introduced:

$$
U_{\text {bend }}=\kappa T \sum\left(1-\frac{\left(\boldsymbol{r}_{i-1}-\boldsymbol{r}_{i}\right)\left(\boldsymbol{r}_{i}-\boldsymbol{r}_{i+1}\right)}{\sigma_{m}^{2}}\right) .
$$

(C) 2001 The American Physical Society

078105-1 
The persistence length of model polymer is calculated as $\lambda_{p} / \sigma_{m} \simeq \kappa-0.5(\kappa>2)$, where $\kappa \simeq 25$ corresponds to the persistence length of uncomplexed natural DNA under usual solution conditions.

We adopted the underdamped Langevin equation for the motion of all monomers and the core particle and neglected the hydrodynamics interaction for simplicity:

$$
\begin{aligned}
& m \frac{d^{2} \boldsymbol{r}_{i}}{d t^{2}}=-\gamma_{m} \frac{d \boldsymbol{r}_{i}}{d t}+\boldsymbol{R}_{m, i}(t)-\frac{\partial U}{\partial \boldsymbol{r}_{i}}, \\
& M \frac{d^{2} \boldsymbol{r}}{d t^{2}}=-\gamma_{p} \frac{d \boldsymbol{r}}{d t}+\boldsymbol{R}_{p}(t)-\frac{\partial U}{\partial \boldsymbol{r}}
\end{aligned}
$$

where $m(M), \gamma_{m}\left(\gamma_{p}\right)$ are the mass and friction constant of the monomer (core particle), respectively. Random force $\boldsymbol{R}_{m, i}(t), \boldsymbol{R}_{p}(t)$ is Gaussian white noise which obeys the fluctuation-dissipation theorem. The internal energy $U$ consists of the four terms described above $U=U_{\text {M.rep }}+$ $U_{M}+U_{\text {bond }}+U_{\text {bend }}$. The ratio of the friction constant between the core particle $\gamma_{p}$ and monomer $\gamma_{m}$ is calculated according to Stokes law. We choose a relatively large mass $m=M\left(0.5 \sigma_{m} / 1.3 \sigma_{m}\right)^{3}=1$ to save calculation time, but this should not affect the motion within the time scale of interest, which is much longer than the relaxation time of velocity. The dynamics of the system is performed using a leapfrog algorithm with time step of $\Delta t=$ $0.005 \tau$, where $\tau=\gamma_{m} \sigma_{m}^{2} / T$ is a unit time step. All simulations were carried out with $3 \times 10^{4} \sim 10^{5}$ time steps.

First, we examined the dependence of the equilibrium structure on the stiffness of the chain. Figure 1(A) shows the snapshots of the conformation of the complex. The left and center images show the proper folding of a stiff chain, $\kappa=10$, where the core is positioned at the chain end and at the chain midpoint, respectively. The right image shows an example of misfolding with a less stiff chain, $\kappa=6$. To evaluate the difference in structure, we introduce the following parameter $\eta$ as a measure of proper ordering:

$$
\eta=\frac{\left|\sum_{\langle i\rangle} \boldsymbol{r}_{i, i+1} \times \boldsymbol{r}_{i+1, i+2}\right|}{N_{p}},
$$

where the summation in $\eta$ is taken only over the monomers in the vicinity of the particle's surface $\left(r_{i}<\sigma+\sigma_{m}\right)$, and $N_{p}$ is the number of the monomers. In the ordered structure, the direction of each vector product between adjacent bond vectors on and/or near the core particle is parallel to the direction of helical axis. Thus, the value of $\eta$ becomes larger from disorder to order in the folded state. Figure 1(B) shows the dependence of the probability distribution of $\eta$ on the chain stiffness. When the chain is stiff enough, the chain always wraps around the particle in an ordered or an helical fashion where $\eta \geq 0.3$ [Fig. 1(A) left, center]. We refer to such proper conformation as a nucleosomelike structure. For stiff chains $(\kappa=8$ and 10 ), only the proper nucleosomelike structure is generated, while the probability of misfolding, $\eta \leq 0.2$ [Fig. 1(A)

(A)

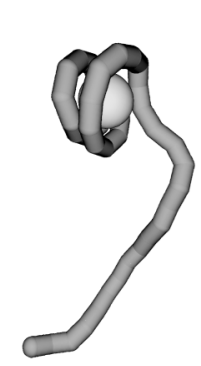

(B)
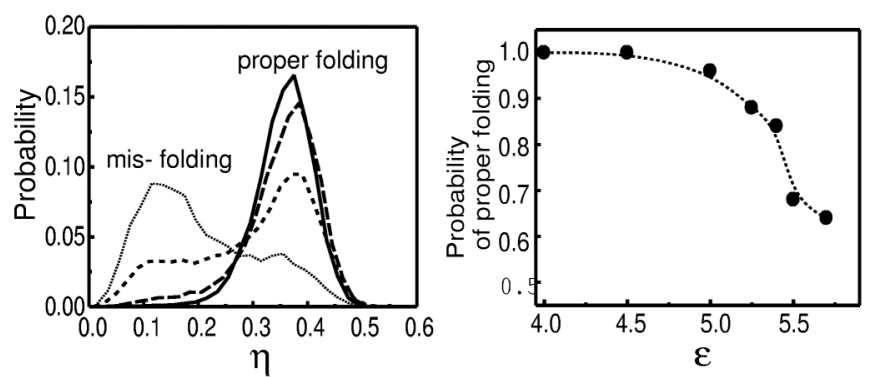

FIG. 1. (A) Snapshots of the complex at a fixed attraction potential, $\epsilon=4$. Left and center images are two representative conformations for a stiff chain of $\kappa=10$, while the right image is an example of misfolding for a less stiff chain of $\kappa=6$. In these conformations, the degree of proper folding, $\eta$, is 0.433 , 0.410 , and 0.143 , respectively. (B) Probability distribution of the order parameter $\eta$ with $\epsilon=4$, and $\kappa=10$ (solid line), $\kappa=8$ (long-dashed line), $\kappa=6$ (short-dashed line), $\kappa=4$ (dotted line). (C) Probability of proper folding, $\eta \geq 0.3$, with $\kappa=10$ dependent on the attraction potential $\epsilon$.

right], increases with a decrease in stiffness. As the chain becomes more flexible, the nucleosomelike structure is less favored, since the entropy gain due to the possible number of states of the chain on the surface dominates the bending energy cost in the flexible chain.

Figure $1(\mathrm{C})$ shows the decrease in the probability of proper folding with an increase in attraction between the chain and core particle for a stiff chain of $\kappa=10$. When the attraction is not too strong $(\epsilon \sim 4)$, the proper nucleosomelike structure is always organized spontaneously during thermal fluctuation. However, if the interaction is too strong, the probability of misfolding $(\eta \leq 0.2)$ increases, since the complex stays in a kinetically trapped conformation. It is well known for experimentalists that the nucleosome structure has failed to be reconstituted through the direct mixing of DNA and histone under physiological salt conditions. According to the standard protocol for reconstitution, DNA and histone are first mixed at a high salt concentration $(2 \mathrm{M} \mathrm{NaCl})$, and the salt concentration is then gradually decreased using salt-gradient dialysis over a period of several hours or days [3]. Our simulation result explains well why this protocol involves such a tedious procedure for reconstituting nucleosomes identical to native ones, considering that the attractive interaction 
between DNA and histone should be rather strong under physiological salt concentration, and, thus, that direct mixing at this concentration leads to misfolding. Even if the probability of misfolding for individual mononucleosomes seems to be small, the frequency to obtain an irregular conformation should increase for multiple nucleosomes on long DNA.

To examine the effect of the polymer end on the positioning of the core particle, we define the relative position of the core particle along the chain $R_{s}$ as

$$
R_{S}= \begin{cases}\frac{N_{b}}{N_{a}+N_{b}} & \left(\text { if } \frac{N_{b}}{N_{a}+N_{b}} \leq 0.5\right) \\ 1.0-\frac{N_{b}}{N_{a}+N_{b}} & \text { (otherwise) }\end{cases}
$$

where $N_{a}$ is the number of monomers at one edge of the chain which is not adsorbed onto the particle, and $N_{b}$ is the same quantity for the other side of the chain. According to this definition, $R_{s}=0$ and $R_{s}=0.5$ correspond to core particles that are positioned on the end and at the center of a polymer chain, respectively. The distribution of $R_{s}$ obtained by the simulation is presented in Fig. 2, which indicates that the core particle prefers to be positioned at the chain end. This phenomenon is a purely entropic effect due to restriction of the polymer chain near the condensed part of the complex (both the core particle and the adjacent turn of the polymer). We consider the conformation of a chain (length $\left.N \sigma_{m}\right) \boldsymbol{r}(s)$ whose one end $(s=0)$ is fixed on the core's surface. By introducing a characteristic length $\lambda$, below which the number of states for the chain segment is suppressed due to the steric effect, the entropy of the chain is calculated as

$$
S(N)=\sum_{n=1}^{N} \log \left[W-\left(W-W_{0}\right) \exp (-n / \lambda)\right],
$$

where $W_{0}$ and $W$ are the number of states for the chain segment next to the core part and its asymptotic value in a noncorrelated region, respectively. The ratio $W / W_{0}$ and

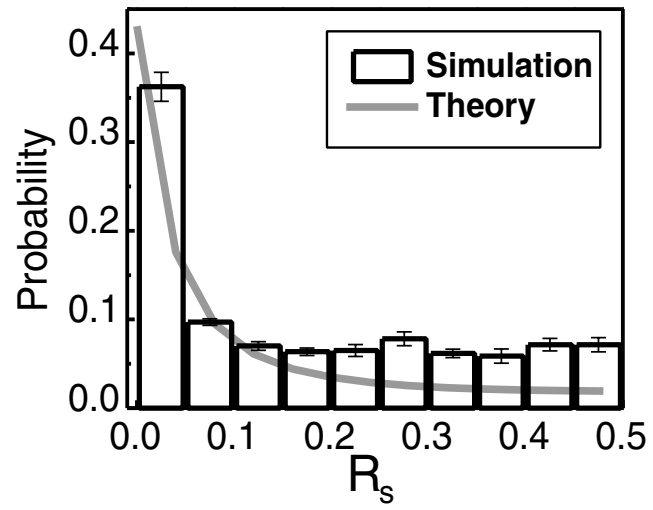

FIG. 2. Probability distribution of the position of the core particle along the chain $R_{s}$. The bar represents the result of a simulation, with $\kappa=10$ and $\epsilon=5.25$. The line is obtained by the calculation described in the text under the following conditions: $W / W_{0}=3.0, \lambda=4.0, N_{a}+N_{b}=25$. $\lambda$ depend on the nature of the interaction, stiffness, and its microscopic mechanism. The probability distribution of $R_{S}$ is calculated as

$$
\begin{aligned}
P\left(R_{S}\right) & \propto \exp \left\{-\frac{\triangle E}{T}+S\left(N_{a}\right)+S\left(N_{b}\right)\right\} \\
& \propto \exp \left\{S\left(N_{a}\right)+S\left(N_{b}\right)\right\},
\end{aligned}
$$

since internal energy does not depend on the position of the core particle along the chain in our homopolymer model. The result is also shown in Fig. 2.

To verify the above notion, nucleosomes were reconstituted from DNA and histone octamer by the salt dialysis method [11], and observed using atomic force microscopy (AFM). Figure 3(A) shows a typical AFM image of reconstituted nucleosomes, where almost all of the DNA form complex with histone core. The size of the core, taking into account the edge effect of the AFM tip [12], is equal to the nucleosome core obtained from x-ray crystal analysis [13]. Figure 3(B) shows the probability distribution of the position of histone on the DNA chain $R_{p}$ based on the AFM observation. Note that, in the definition of $R_{p}$, the distance between the center of the nucleosome core

(A)

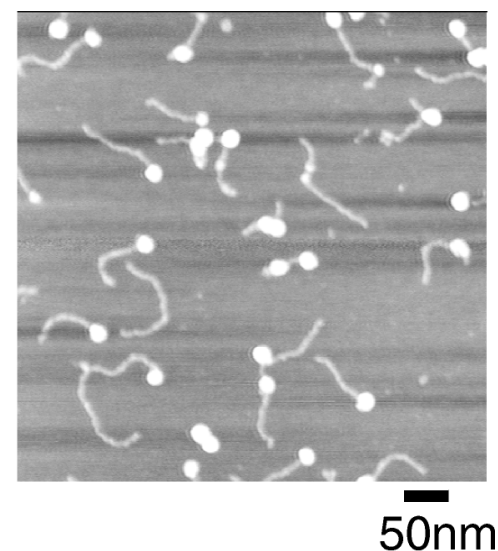

(B)

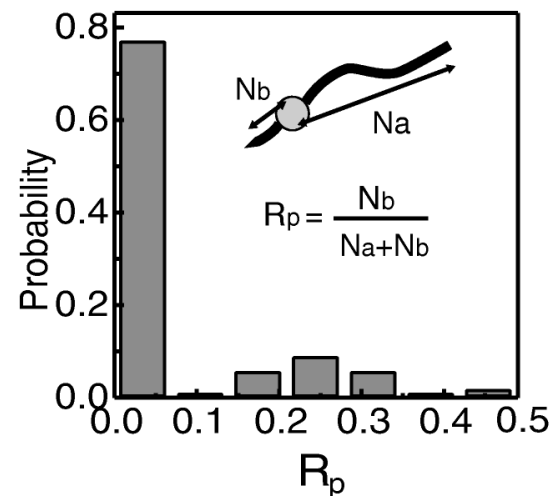

FIG. 3. (A) Atomic force microscopy image of reconstituted nucleosome from a 437 bp DNA chain. (B) Probability distribution of the position of histone on the DNA chain. $R_{p}$ is measured from the AFM image as shown schematically in the figure. 
and the chain end is used for $N_{a}$ or $N_{b}$, which are used in the definition of $R_{S}$. These figures show that the histone core is positioned at the chain end with a very high probability, indicating that the end effect is significant in nucleosomes. It is noted that some previous studies using the gel electrophoresis technique have also suggested that DNA end has some effects on the positioning of histone more or less, which depend on the base pair sequence and the length of DNA [14]. Now the origin of this end effect has become clear; we can conclude that it is not a special phenomenon depending on the materials, but rather is a general feature inherent in the nucleosomelike structure. Therefore, it is important to take into account this end effect of the chain, especially when considering the inherent properties of short chromatin fibers (e.g., mono-, di-, and oligonucleosomes).

It may be important to note that nucleosomes are not static at all under equilibrium; in fact, the position of the core particle fluctuates relative to the chain in a stochastic manner by sliding along the chain. This dynamic behavior can be considered as one manifestation of quasi-onedimensional Brownian motion. Interestingly, during this process, the chain does not unfold from the core particle, but remains in a wrapped manner around it. Calculation of the mean square displacement of the core particle along the chain (where we used circular chain with length $N=100$ to remove the edge effect) in a nucleosomelike structure shows that the effective diffusion coefficient of the core particle along the chain depends on the effective interaction between the core particle and the chain; the mobility decreases monotonously with an increase in $\epsilon$ or the number of monomers interacting with the core particle. In an actual nucleosome, the situation is more complicated; free energy as a function of the position of the core particle along the chain has a complicated shape which depends on both of the DNA sequence and its surroundings. Recent studies on the nucleosome structure using two-dimensional electrophoresis suggest that the histone core protein is mobile with regard to the DNA chain, which is referred to as nucleosome sliding $[11,14,15]$. Contrary to this, it is often claimed that nucleosome sliding might not be an actual event but a consequence of the site exposure, in which a segment of DNA released from the histone surface can loop back and be recaptured at a new position, creating a nucleosome with a "bulged" loop of DNA [16]. Thus, the problem on the nucleosome dynamics is still under dispute. Our simulation indicates that, even when the core particle is bound with an energy of tens of $k_{B} T$, the sliding motion along the chain is left as a survived freedom in the nucleosomelike structure. Such a motional freedom demonstrates a possible mechanism to expose the recognition sites for the regulatory proteins [17]. This freedom may also affect the process of the organization of the higher order structure of chromatin.

At the length scale of nucleosomes (on the order of the persistence length), the effect of the local structure such as twisting [18,19] and base pair sequence variations [20-22] should strongly affect the structure and function of the nucleosome. For example, the degree of twisting should have an important effect on the direction of wrapping around histone, i.e., in a counterclockwise fashion. Direct inclusion of the Coulomb interaction on the model system would also give some additional insight on the stability of the nucleosome structure $[6,8]$. Considering such effects in addition to the general properties obtained in this study, it may become possible to obtain the underlying mechanism of the self-regulation of gene expression in relation to the structural dynamics of chromatin in living cells.

This study was partly performed through Special Coordination Funds of the Ministry of Education, Culture, Sports, Science and Technology, the Japanese Government.

[1] A. Wolffe, Chromatin Structure and Function (Academic, London, 1998), 3rd ed.

[2] R. D. Kornberg and Y. Lorch, Cell 98, 285 (1999).

[3] K. E. van Holde, Chromatin (Springer, New York, 1988).

[4] N. L. Marky and G. S. Manning, J. Mol. Biol. 254, 50 (1995).

[5] T. Wallin and P. Linse, Langmuir 12, 305 (1996).

[6] E. M. Mateescu, C. Jeppesen, and P. Pincus, Europhys. Lett. 46, 493 (1999).

[7] R. R. Netz and J.-F. Joanny, Macromolecules 32, 9026 (1999).

[8] K.-K. Kunze and R. R. Netz, Phys. Rev. Lett. 85, 4389 (2000).

[9] H. Schiessel, J. Rudnick, R. Bruinsma, and W. M. Gelbart, Europhys. Lett. 51, 237 (2000).

[10] H. Noguchi and K. Yoshikawa, J. Chem. Phys. 113, 854 (2000).

[11] K. Ura, J. Hayes, and A.P. Wolffe, EMBO J. 14, 3752 (1995).

[12] S. Nettikadan, F. Tokumasu, and K. Takeyasu, Biochem. Biophys. Res. Commun. 226, 645 (1996).

[13] K. Luger, A. W. Mader, R. K. Richmond, D. F. Sargent, and T. J. Richmond, Nature (London) 389, 251 (1997).

[14] G. Meersseman, S. Pennings, and E. M. Bradbury, EMBO J. 11, 2951 (1992).

[15] S. Pennings, G. Meersseman, and E. M. Bradbury, J. Mol. Biol. 220, 101 (1991).

[16] J. Widom, Annu. Rev. Biophys. Biomol. Struct. 27, 285 (1998).

[17] J. L. Workman and R.E. Kingston, Annu. Rev. Biochem. 67, 545 (1998).

[18] Yu.S. Velichko, K. Yoshikawa, and A. R. Khokhlov, J. Chem. Phys. 111, 9424 (1999).

[19] I. Tobias, D. Swigon, and B. D. Coleman, Phys. Rev. E 61, 747 (2000); 61, 759 (2000).

[20] C. Anselmi, G. Bocchinfuso, P. D. Santis, M. Savino, and A. Scipioni, Biophys. J. 79, 601 (2000).

[21] T. E. Shrader and D. M. Crothers, Proc. Natl. Acad. Sci. U.S.A. 86, 7418 (1989).

[22] P. T. Lowary and J. Widom, Proc. Natl. Acad. Sci. U.S.A. 94, 1183 (1997). 